\title{
AVALIAÇÃO DE MÉTODOS PARA QUANTIFICAÇÃO DE CHUVA DIRIGIDA NAS FACHADAS DAS EDIFICAÇÕES
}

\section{EVALUATION OF METHODS FOR MEASURING DRIVING RAIN ON BUILDINGS FACADES}

\author{
Vanda Alice Garcia Zanoni ${ }^{1}$ \\ Universidade de Brasília, Brasília, DF, Brasil, vandazanoni@unb.br \\ José Manoel Morales Sánchez ${ }^{2}$ \\ Universidade de Brasília, Brasília, DF, Brasil, sanchez@unb.br \\ Elton Bauer 3 \\ Universidade de Brasília, Brasília, DF, Brasil, elbauerlem@gmail.com
}

\section{Resumo}

A chuva dirigida é uma das principais fontes de umidade das fachadas das edificações, desencadeando mecanismos de degradação que envolvem processos de molhagem e secagem, variações higrotérmicas e perda de estanqueidade dos constituintes. Existem diversos métodos de quantificação de chuva dirigida para calcular a intensidade de água que é projetada na fachada. Esses métodos permitem avaliar as condições de exposição que afetam a durabilidade e vida útil das edificações, assim como a intensidade da ação deste fenômeno. As normas internacionais ISO 15927-3: 2009 e ASHRAE Standard 160: 2009 são exemplos de documentos de referência que fornecem procedimentos e critérios para estimar a quantidade de chuva dirigida que se projeta na superfície vertical, em cada orientação de fachada. No cenário nacional, as normas brasileiras não apresentam critérios, modelos ou métodos de cálculo para a quantificação de chuva dirigida. Considerando as lacunas existentes no contexto brasileiro, este artigo apresenta métodos e modelos identificados por meio de uma revisão de literatura. O foco deste artigo é a avaliação dos principais métodos selecionados para quantificação da chuva dirigida em fachada, embasada em estudos descritivos e analíticos que permitiram a comparação entre eles. Os resultados mostram que os métodos semi-empíricos avaliados resultam em diferentes montantes de chuva dirigida, visto que os métodos adotam diferentes parâmetros para os coeficientes que retratam as condições de exposição do edifício e sua inserção no contexto urbano.

Palavras-chave: Condições de exposição. Chuva dirigida. Umidade. Degradação.

\begin{abstract}
Directed rain is one of the primary sources of humidity in building façades, triggering degradation mechanisms involving wetting and drying processes, hygrothermal variations and leakage of constituents. There are several methods of quantification of directed rain to calculate the intensity of water that is projected on the facade. These methods allow evaluating the exposure conditions that affect the durability and useful life of the buildings, as well as the intensity of the action of this phenomenon. The ISO 15927-3: 2009 and ASHRAE Standard 160: 2009 international standards are examples of reference documents that provide procedures and criteria for estimating the amount of directed rainfall projected on the vertical surface in each façade orientation. In the national scenario, Brazilian standards do not present criteria, models or calculation methods for the quantification of directed rainfall. This article presents methods and models, identified through a literature review, aiming to fill the gap in the Brazilian context. The focus of this article is the evaluation of the primary methods selected for the quantification of directed rain on the façade, based on descriptive and analytical studies that allowed the comparison between them. The results show that the semi-empirical methods evaluated result in different amounts of directed rain since the methods adopt different parameters for the coefficients that portray the conditions of exposure of the building and its insertion in the urban context.
\end{abstract}

Keywords: Exposure conditions. Driving rain. Moisture. Degradation.

How to cite this article:

ZANONI, Vanda Alice Garcia; SANCHEZ, José Manoel Morales; BAUER, Elton. Métodos para quantificação de chuva dirigida incidente nas fachadas das edificações. PARC Pesquisa em Arquitetura e Construção, Campinas, SP, v. 9, n. 2, p. 122-132, jun. 2018. ISSN 1980-6809. Disponivel em: <https://periodicos.sbu.unicamp.br/ojs/index.php/parc/article/view/8650260>. Acesso em: 19 maio 2018. doi:https://doi.org/10.20396/parc.v9i2.8650260. 


\section{Introdução}

A chuva dirigida (driving rain ou wind-driven rain WDR) é uma das principais fontes de umidade das edificações. Decorrente da ação do vento sobre a precipitação, a chuva dirigida é projetada na superfície da envoltória edificada, desencadeando mecanismos de degradação que envolvem processos de molhagem e secagem, variações higrotérmicas nos materiais porosos e perda da estanqueidade dos constituintes das fachadas.

Existem diversos métodos de quantificação de chuva dirigida para calcular a intensidade de água que é projetada na fachada, no instante antes do impacto das gotas de chuva sobre a superfície vertical (BLOCKEN et al., 2013). Esses métodos permitem avaliar as condições de exposição que afetam a durabilidade e vida útil das edificações, assim como a intensidade da ação deste fenômeno na relação dose e resposta do edifício, após o impacto das gotas de chuva.

As normas internacionais ISO 15927-3 (BSI, 2009), Standard 160 (ANSI/ASHRAE, 2009) e ISO 15686-7 (BSI, 2006) são alguns exemplos de documentos de referência que fornecem procedimentos e critérios para estimar a quantidade de chuva dirigida que se projeta na superfície vertical, em cada orientação de fachada. No cenário nacional, as normas brasileiras não apresentam critérios, modelos ou métodos de cálculo para a quantificação de chuva dirigida.

Visando preencher a lacuna de conhecimento existente no contexto brasileiro quanto à intensidade de chuva dirigida em fachadas e seu comportamento nas diferentes condições de exposição, este artigo apresenta métodos e modelos identificados por meio de uma revisão de literatura.

O objetivo deste artigo é avaliar os principais métodos selecionados para quantificação da chuva dirigida em fachada, embasando-se em estudos descritivos e analíticos que permitem a comparação entre eles.

\section{Fundamentação}

Para avaliar a chuva nas fachadas (instante antes do impacto das gotas de chuva), os estudos de quantificação de chuva dirigida são realizados a partir de três métodos: (1) métodos experimentais (medições in situ ou em laboratório), (2) métodos semi-empíricos, e (3) métodos numéricos - simulações numéricas baseadas em Dinâmica dos Fluidos Computacional (Computational Fluid Dynamics - CFD) (BLOCKEN; CARMELIET, 2010). O termo semi-empírico refere-se aos métodos que usam equações de cálculo embasadas teoricamente na física das construções, e com uso de coeficientes que são determinados a partir de medições experimentais in situ.
$\mathrm{O}$ conceito de chuva dirigida foi apresentado e consolidado, durante os anos 60, pelo CIB (International Council for Research and Innovation in Building and Construction). Esse conceito foi baseado nas pesquisas de Lacy (1965) que fundamentou o seu trabalho no primeiro mapeamento de chuva dirigida elaborado por Hoppestad (1955) para a Noruega. Baseado em uma relação entre o vento e a precipitação, o mapeamento da chuva nos edifícios tinha como objetivo tipificar soluções construtivas para paredes, face à severidade da zona climática em que os edifícios eram construídos (LACY; SHELLARD,1962; FREITAS, 2011; KRPAN, 2013).

Lacy (1965) propôs o conceito do Índice de Chuva Dirigida (ICD ou DRI - Driving Rain Index) como sendo o produto da precipitação pela velocidade do vento incidente nas edificações, expresso por médias anuais conforme apresentado pela Equação (1).

$$
I C D=V \cdot \frac{P}{1000}
$$

Onde: ICD - Índice de chuva dirigida $\left(\mathrm{m}^{2} / \mathrm{s}\right) ; \mathrm{V}$ Velocidade média do vento $(\mathrm{m} / \mathrm{s}$, medida a $10 \mathrm{~m}$ de altura); P - Precipitação anual (mm).

Em relação ao nível de exposição à chuva dirigida, Lacy (1972) propôs três faixas de classificação: Protegido para $\mathrm{ICD} \leq 3$; Moderado para $3<\mathrm{ICD} \leq 7$ e Severo para ICD $>$ 7. A classificação dada por Lacy (1972) não considerou nenhum aspecto do entorno próximo à edificação ou da própria edificação.

Chand e Bhargava (2002) ampliaram a classificação de Lacy (1972) para quatro faixas de exposição (Quadro 1). Marsh (1977) propôs correções para os valores apresentados por Lacy (1972), em função da proximidade de regiões costeiras, classificando as áreas distantes até 8 $\mathrm{km}$ do mar ou de grandes estuários, com ICD $\leq 3 \mathrm{~m}^{2} / \mathrm{s}$, como exposição moderada, e para $3<\mathrm{ICD} \leq 7$ como áreas de exposição severa, conforme apresentado no Quadro 1 (SILVA; GIRALT, 1995; LIMA; MORELLI, 2005; GIONGO, 2007).

Quadro 1 - Nível de exposição à chuva dirigida
\begin{tabular}{|c|c|c|c|}
\hline $\begin{array}{c}\text { Indice de } \\
\text { Chuva Dirigida } \\
\left(\mathrm{m}^{2} / \mathbf{s}\right)\end{array}$ & Lacy (1972) & $\begin{array}{c}\text { Chand } \mathrm{e} \\
\text { Bhargava } \\
(2002)\end{array}$ & $\begin{array}{c}\text { Marsh }(1977) \\
\text { Recomendação }\end{array}$ \\
\hline$I C D \leq 3$ & Protegido & Protegido & Moderado \\
\hline $3<I C D \leq 5$ & Moderado & Moderado & Moderado \\
\hline $5<I C D \leq 7$ & Moderado & Moderado & Severo \\
\hline $7<I C D \leq 11$ & Severo & Alto & Severo \\
\hline$I C D>11$ & Severo & Severo & Severo \\
\hline
\end{tabular}

Fonte: Adaptado de Giongo, Padaratz,e Lamberts (2011).

O ICD é um índice indicativo das condições de exposição do edifício, considerando uma situação em campo aberto, onde o vento não sofre nenhuma perturbação em sua linha de fluxo. Nesse caso, para aplicar a Equação 1, são 
utilizados valores médios anuais de precipitação e de direção e velocidade do vento.

O ICD como um valor anual, calculado pelas médias anuais de precipitação e vento, pode mascarar o comportamento sazonal dos agentes climáticos envolvidos no fenômeno e o nível de exposição da envoltória da edificação de acordo com a sua orientação. Por isso, alguns autores calculam o ICD a partir de valores mensais de precipitação e vento, inclusive considerando índices para cada direção de vento (índice direcional). Como resultado, obtém-se índices de chuva dirigida direcional, para cada mês do ano (GIONGO, 2007; ESTRELA, 2010; MELO JÚNIOR; CARASEK, 2011; ZANONI et al., 2014).

Segundo Krpan (2013), Lacy (1972) reconheceu que os índices de chuva dirigida (ICD) não eram uma indicação direta da quantidade de chuva que realmente era projetada na parede de um edifício, devido às perturbações no fluxo de ar influenciadas pelos edifícios. Para Lacy (1972), as condições médias anuais assumidas no cálculo do índice de chuva dirigida não representavam efetivamente as condições mais severas das chuvas. Este autor concluiu, no entanto, que a elaboração de mapas de chuva com base nos índices era válida, podendo ser usados por projetistas, arquitetos e pesquisadores como uma indicação da gravidade da exposição para um determinado local de construção.

O conceito do índice de chuva dirigida de Lacy (1972) tornou-se a base para os métodos analíticos (semiempíricos) de quantificação de chuva dirigida que levam em consideração parâmetros relacionados às características do edifício e do entorno edificado, tais como topografia, rugosidade, obstruções, tipologia e altura da fachada. Justifica-se assim os estudos descritivos e analíticos dos principais métodos semi-empíricos para quantificação da chuva dirigida em fachada, identificados na revisão de literatura.

\section{Métodos semi-empíricos}

A partir do Índice de Chuva Dirigida (ICD), Lacy (1965) estendeu sua abordagem para estabelecer relações teóricas entre a intensidade da chuva projetada na superfície vertical (Rv) e a intensidade da precipitação caindo em superfície horizontal (Rh), assumindo gotas de diâmetro médio, distribuição uniforme e velocidade terminal de 4,5 $\mathrm{m} / \mathrm{s}$, o que corresponde a um diâmetro de gota de chuva de 1,2 $\mathrm{mm}$ (adequadas para chuvas de intensidade média) (BLOCKEN; CARMELIET, 2004). A expressão de cálculo resultante é demonstrada na Equação (2).

$$
R_{v}=R_{h} \cdot \frac{U}{v t}
$$

$$
\begin{gathered}
R_{v}=R_{h} \cdot \frac{U}{4,505 \cdot R_{h}^{0,123}} \\
R_{v}=0,222 \cdot \mathrm{U} \cdot R_{h}^{0,88} \approx 0,222 \cdot \mathrm{U} \cdot R_{h}
\end{gathered}
$$

Onde: $R_{v}$ - Intensidade de chuva dirigida (mm/h ou $\left.1 / \mathrm{h} . \mathrm{m}^{2}\right)$; $R_{h}$ - Intensidade da precipitação em superfície horizontal $\left(\mathrm{mm} / \mathrm{h}\right.$ ou $\left.1 / \mathrm{h} . \mathrm{m}^{2}\right) ; U$ - Velocidade do vento $(\mathrm{m} / \mathrm{s})$ e $v t-$ Velocidade terminal da gota de chuva $(\mathrm{m} / \mathrm{s})$.

A expressão de cálculo proposta por Lacy (Equação 2) considera a intensidade de chuva dirigida em uma situação de campo aberto. $\mathrm{O}$ coeficiente de proporcionalidade de Lacy $(\mathrm{k}=0,222 \mathrm{~s} / \mathrm{m})$ é denominado por Blocken $\mathrm{e}$ Carmeliet (2004) como coeficiente de chuva dirigida em campo aberto (free driving rain coefficient).

A Equação 2 assume que a direção do vento é sempre perpendicular à superfície vertical. Observa-se que a expressão final foi simplificada, desconsiderando o expoente. Segundo Blocken e Carmeliet (2004), o expoente 0,88 , em boa aproximação, pode ser omitido.

Em geral, para a quantificação da intensidade de chuva dirigida na superfície vertical, a Equação 2 passou a ser representada pela Equação 3, que considera a precipitação associada a cada velocidade e direção de vento. Dessa forma, são contempladas tanto a chuva passível de atingir a fachada como as condições locais.

Segundo Blocken et al. (2011), o valor do coeficiente de chuva dirigida $\alpha$ é o que distingue os diferentes métodos de quantificação. Dependendo das condições de exposição e do edifício, o coeficiente $\alpha$ da Equação (3) pode adquirir diferentes expressões, conforme o método adotado para o cálculo.

$$
R_{w d r}=\propto \cdot V \cdot P \cdot \cos (D-\theta)
$$

Onde: $R_{w d r}$ - Intensidade de chuva dirigida $\left(1 / \mathrm{m}^{2}\right) ; \alpha-$ Coeficiente de chuva dirigida; V - Velocidade do vento tomada a $10 \mathrm{~m}$ de altura $(\mathrm{m} / \mathrm{s}) ; \mathrm{P}$ - Precipitação em superfície horizontal (mm); $\Theta$ - Orientação da fachada: ângulo entre o Norte e a direção normal à parede $\left(^{\circ}\right)$ e $\mathrm{D}$ Direção do vento (ângulo a partir do Norte) $\left({ }^{\circ}\right)$.

\section{Estado da arte dos estudos brasileiros sobre chuva dirigida}

Entre os estudos pioneiros de chuva dirigida no Brasil, destacam-se os seguintes trabalhos: (1) Perez (1986) estudou os tipos de umidade nas edificações; (2) Bauer (1987) analisou os fenômenos que envolvem a penetração de água de chuva em fachadas e o grau de exposição das fachadas frente à ação da chuva dirigida; e (3) Kazmierczak (1989) estudou a influência da quantidade de chuva dirigida nos problemas existentes nas alvenarias de tijolos. 
Usando métodos semi-empíricos, Silva e Giralt (1995) estabeleceram o ICD anual para a cidade de Porto Alegre a partir de dados horários de precipitação, velocidade e direção do vento de cinco anos (1977- 1981).

Lima e Morelli (2005) realizaram o primeiro mapa de chuva dirigida para o Brasil, com dados do Índice de Chuva Dirigida do ano de 2004. A metodologia adotada considerou o total de chuva anual e a média anual de vento e determinou um mapa que traz informações sobre o grau de exposição, apenas para o ano de 2004, sem indicações de direção predominante da incidência de chuva dirigida.

Lima e Estrela (2010) determinaram os Índices de Chuva Dirigida para a cidade de São José dos Campos - SP, por meio da análise de dados de um ano de monitoração. Estrela (2010) também determinou o ICD para 400 localidades correspondentes às estações meteorológicas brasileiras, inclusive Brasília, considerando os dados sinóticos diários da série 1995-2010 da base de dados do CPTEC/INPE. Para o cálculo do ICD, os autores utilizaram as médias mensais do vento e a precipitação acumulada mensal. Os valores dos ICD mensais obtidos não são direcionais, isto é, não identificam direções de ventos predominantes ou orientações de fachadas mais impactadas.

Melo Júnior e Carasek (2011) determinaram os Índices de Chuva Dirigida direcionais mensais para a cidade de Goiânia-GO, considerando sete anos de dados meteorológicos, no período de 2002 a 2008.

Giongo (2007) e Giongo, Padaratz e Lamberts (2011) apresentaram os Índices de Chuva Dirigida direcionais mensais para a cidade de Florianópolis-SC, com base na análise de três períodos: 1961-1970, 1991/1993-1996 e setembro de 2005 a agosto de 2006.

Zanoni et al. (2014) publicaram uma Matriz de Análise dos Índices de Chuva Dirigida direcionais mensais para a cidade de Brasília, considerando três séries históricas de dados meteorológicos consolidados (1960-1990; 19912000; 2001-2010).

Durante a revisão de literatura, constatou-se que até 2014 os pesquisadores brasileiros buscavam a determinação do ICD como índice representativo das condições de exposição. No entanto, estudos referentes à aplicação de métodos para quantificação de chuva dirigida considerando o contexto urbano foram encontrados nos trabalhos dos pesquisadores Zanoni, Sánchez e Bauer (2014) que publicaram um estudo para quantificação da intensidade de chuva dirigida para a cidade de BrasíliaDF, aplicando o método semi-empírico da norma ISO 15927-3 (2009).

Pesquisas experimentais in situ para quantificação de montantes de chuva dirigida, em contexto urbano no
Brasil, foram realizadas por Zanoni (2015), com medições efetuadas na cidade de Brasília-DF.

\section{Procedimentos metodológicos}

\section{Seleção dos Métodos}

Por meio da revisão da literatura, foram selecionados três métodos semi-empíricos para quantificação de chuva dirigida: ISO 15927-3:2009, ASHRAE 160:2009, e o método do programa higrotérmico computacional WUFI Pro 5.3 (IBP, 2013). São métodos que levam em consideração as características do edifício, assim como o contexto urbano em que está inserido.

A seleção dos métodos foi baseada nos seguintes trabalhos:

a) Freitas (2011): comparou diversos métodos de quantificação da intensidade da chuva dirigida para Portugal, para fundamentar as análises de sensibilidade para diferentes configurações de fachadas, considerando a influência da chuva dirigida, utilizando o programa computacional de simulação higrotérmica WUFI Pro 5.3 (IBP, 2013).

b) Blocken e Carmeliet (2004): compararam três modelos de avaliação de chuva dirigida, considerando que o método semi-empírico apresentado pela ISO 15927-3:2009 pode ser considerado um dos mais utilizados e avançados.

c) Straube (2010): aplicou os métodos da ASHRAE 160:2009 e do WUFI em estudos sobre a previsão simplificada de incidência da chuva dirigida.

O método padronizado pela norma internacional ISO 15927-3:2009 é um método originado da BS 8104:1992 (BSI, 1992). Inclusive, esse método é recomendado pela ISO 15686-7 (BSI, 2006) para a avaliação das condições do clima local e do microclima e para expressar a provável quantidade de água que impacta a parede em uma determinada orientação.

O programa de simulação higrotérmica WUFI Pro 5.3 (IBP, 2013) apresenta o método desenvolvido e validado pelo Instituto Fraunhofer. Como opção para os dados de entrada, o programa também oferece ao utilizador o método de quantificação da chuva dirigida preconizado pela ASHRAE 160:2009.

\section{Descrição do método da ISO 15927-3: 2009}

A ISO 15927-3 (2009) permite calcular a provável quantidade de água de chuva dirigida em uma fachada, em uma dada orientação, e considera os dados climáticos horários de precipitação e vento (velocidade e direção), de um determinado período de tempo, onde o vento sopra na direção da fachada. 
A Equação (4), apresentada pela ISO 15927-3:2009, estabelece a intensidade de chuva dirigida em um determinado período de tempo em campo aberto para uma determinada orientação de fachada $(\theta)$, considerando todos os eventos horários de precipitação e vento que sopra em direção (D) à fachada.

$$
I_{\mathrm{S}}=\frac{2}{9} \sum V P^{8 / 9} \cos (D-\theta)
$$

Onde: $I_{s}$ - Intensidade de chuva dirigida em uma determinada orientação de fachada $(\Theta)$, em um determinado período em campo aberto $\left(1 / \mathrm{m}^{2}\right) ; V$ - Média horária da velocidade do vento $(\mathrm{m} / \mathrm{s})$, a dez metros de altura; $P$ - Precipitação acumulada horária em superfície horizontal (mm); $\theta$ - Orientação da fachada: ângulo entre o Norte e a direção normal à parede e $D$ - Direção do vento (ângulo a partir do Norte).

$\mathrm{O}$ valor Is obtido na Equação 4 representa a intensidade em campo aberto. Esse valor deve ser aplicado à Equação 5 para que se obtenha a intensidade de chuva dirigida no contexto urbano.

A Equação (5) leva em consideração a intensidade de chuva dirigida na fachada sujeita às condições de exposição da edificação (topografia, rugosidade, obstruções e tipologia da fachada).

$$
I_{W S}=I_{S} C_{R} C_{T} O W
$$

Onde: $I_{w s}$ - Intensidade de chuva dirigida em uma determinada orientação de fachada $(\Theta)$, em um determinado período e contexto urbano $\left(1 / \mathrm{m}^{2}\right) ; \mathrm{Cr}$ Coeficiente de rugosidade; CT - Coeficiente de topografia; $\mathrm{O}$ - Fator de obstrução e W - Fator da fachada.

A ISO 15927-3:2009 oferece uma série de tabelas com critérios e parâmetros para que o utilizador possa selecionar os mais adequados para a situação em estudo.

O parâmetro Cr considera a variação da velocidade média do vento devido à altura acima do solo e a rugosidade do terreno na direção em que sopra o vento; dependendo da categoria do terreno.

O fator CT considera o aumento da velocidade média do vento em colinas isoladas e escarpas. Deve ser considerado para locais que estejam a mais de meia altura de uma colina ou para locais que estejam situados até 1,5 vezes a altura de uma escarpa a partir da base da mesma.

$\mathrm{O}$ fator $\mathrm{W}$ é uma forma de considerar a tipologia de fachada (altura e características) e a variação da chuva dirigida ao longo da fachada devido ao fluxo de ar nos pontos singulares. Este fator é dado para as diferentes regiões de fachada.

O fator O considera a proteção da fachada pelo obstáculo mais próximo, que pode ser um edifício, vedações ou árvores. Seu valor é determinado pelo cálculo da distância horizontal ao obstáculo mais próximo que deve ter, no mínimo, a altura da fachada em análise.

\section{Descrição do método da ASHRAE 160:2009}

A ANSI/ASHRAE Standard 160 (2009) trata dos critérios de projeto para controle da umidade em edifícios. A norma apresenta um método para determinar a intensidade de chuva dirigida na fachada.

Para calcular a carga de chuva que atinge uma superfície vertical é dada a Equação (6).

$$
R_{b v}=F e \cdot F d \cdot F c \cdot V \cdot \cos (D-\theta) \cdot R h
$$

Onde: $R_{b v}$ - Deposição da chuva no plano vertical da parede, $\mathrm{mm} / \mathrm{m}^{2}$.h; $R h$ - Intensidade da precipitação, plano horizontal, $\mathrm{mm} / \mathrm{h} ; V$ - Média horária da velocidade do vento a $10 \mathrm{~m}$ de altura, $\mathrm{m} / \mathrm{s} ; \theta$ - Ângulo entre a direção do vento e a normal à parede; $\mathrm{Fe}$ - Fator de exposição à chuva; $F d$ - Fator de deposição da chuva e $F c$ - Constante empírica: $0,2 \mathrm{~s} / \mathrm{m}$.

O fator de exposição Fe é influenciado pela topografia, isto é, depende das características do terreno ao redor do edifício e da altura do edifício. Para o fator de deposição Fd são dados parâmetros considerando três diferentes situações para as regiões de fachada: a) protegidas por beirais; b) não protegidas; c) não protegidas e sujeitas a escorrimentos.

\section{Descrição do método do WUFI PRO 5.3: 2013}

A chuva dirigida é um dos parâmetros de entrada para a simulação higrotérmica pelo programa computacional WUFI Pro 5.3 (IBP, 2013). O programa obtém os dados horários de precipitação, velocidade e direção do vento de um arquivo climático do tipo TMY com 8760 horas, fornecido para alimentar as simulações computacionais. Este programa utiliza a Equação (7) (IBP, 2013).

$$
R_{w d r}=R_{2} \cdot R_{h} \cdot V \cdot \cos (D-\theta)
$$

Onde: $R_{w d r}$ - Intensidade de chuva dirigida $(\mathrm{mm} / \mathrm{h}) ; R_{h}$ Precipitação em superfície horizontal $(\mathrm{mm}) ; R_{2} \quad-$ Coeficiente dependente da localização na fachada $(\mathrm{s} / \mathrm{m}) ; V$ - Média horária da velocidade do vento a $10 \mathrm{~m}$ de altura, $(\mathrm{m} / \mathrm{s}) ; D$ - Direção do vento (ângulo a partir do Norte) e $\theta$ - Orientação da fachada: ângulo entre o Norte e a direção normal à parede.

Os parâmetros $\mathrm{Rh}, \mathrm{V}, \mathrm{D}$, e $\Theta$ são lidos do arquivo climático fornecido como dado de entrada. São dadas opções para a seleção do coeficiente R2 que está relacionado à altura do edifício e à localização da área de estudo na fachada.

O WUFI Pro 5.3 (IBP, 2013) utiliza a Equação 7 para gerar um gráfico ilustrando o montante anual de chuva dirigida para cada orientação de fachada. Este programa permite que o utilizador selecione uma entre duas opções de 
método: o seu próprio método (Equação 7) ou o método estabelecido pela ASHRAE 160:2009 (Equação 6).

Uma das condições de entrada de dados do programa WUFI Pro 5.3 (IBP, 2013) são os coeficientes de transferência à superfície. No caso da chuva dirigida, o utilizador deve selecionar um fator de redução da chuva dirigida entre as seguintes opções: valor definido pelo utilizador; superfície sem absorção; valor dependente da inclinação do elemento construtivo. Para o elemento construtivo cuja inclinação da superfície varia de 0o até 90o, o fator de redução da chuva dirigida é 1, ou seja, é considerada toda a carga de água que incide na superfície (por exemplo, telhado ou paredes inclinadas).

Para superfícies com inclinação de 90o (por exemplo, fachada vertical), o fator de redução é 0,7 , ou seja, é considerada somente $70 \%$ da chuva que incide na superfície, pois leva-se em conta que parte da chuva dirigida é refutada devido aos fenômenos que ocorrem no instante de contato da gota com a superfície (respingo, ressalto, etc.). Portanto, independentemente do método de cálculo selecionado (método WUFI Pro 5.3:2013 ou método ASHRAE 160:2009), o programa considera um coeficiente de transferência à superfície de fachada de $70 \%$ para o montante de chuva dirigida calculado por meio do método selecionado.

\section{Contextualização e critérios para aplicação dos métodos semi-empíricos selecionados}

Para contextualizar o estudo, foi considerado um edifício residencial com seis pavimentos, localizado em uma superquadra no Plano Piloto, ao longo do Eixo Rodoviário Residencial de Brasília. A Figura 1 ilustra o contexto urbano analisado.

Figura 1 - Situação geográfica do edifício na superquadra do Plano Piloto de Brasília

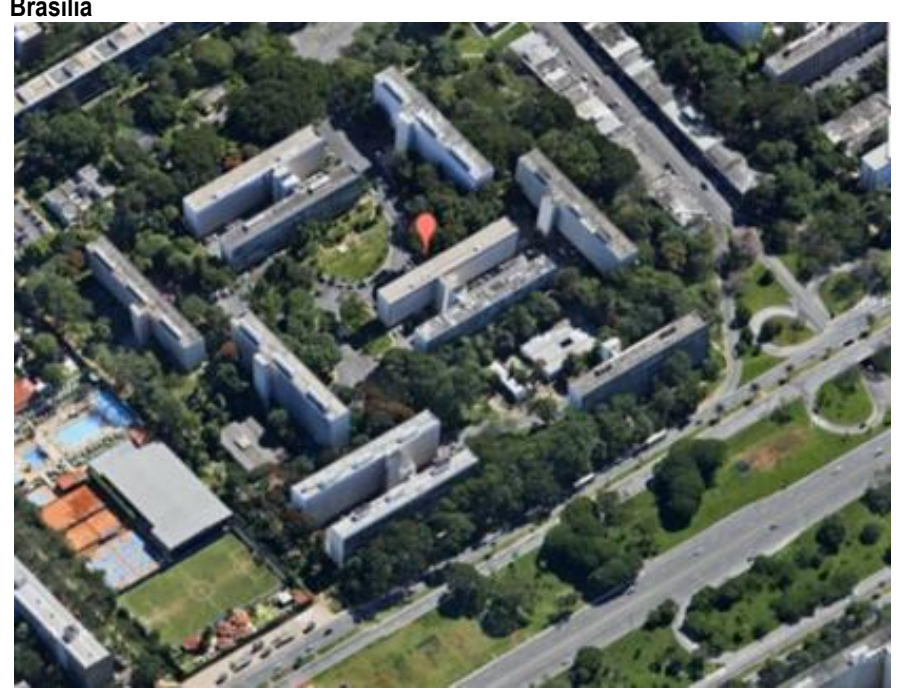

Fonte: http://maps.google.com.br/maps (2017).
Ao selecionar os parâmetros de cada método, teve-se o cuidado de adotar valores representativos das mesmas condições urbanas e da arquitetura do edifício, para que os resultados pudessem ser comparados.

Para a quantificação de chuva dirigida nas fachadas pelos métodos da ASHRAE 160:2009 e da ISO 15927-3:2009, foram considerados todos os dados horários de precipitação, simultaneamente associados aos dados horários de velocidade e direção dos ventos incidentes nos octantes (apresentados no Quadro 2), possibilitando assim a análise da chuva dirigida em cada mês do ano, para as oito principais orientações de fachada correspondentes aos pontos cardeais (Norte, Leste, Sul, Oeste) e aos pontos colaterais (Nordeste, Sudeste, Sudoeste e Noroeste).

Quadro 2 - Orientação de fachada e respectivos octantes

\begin{tabular}{|c|c|}
\hline Orientação da fachada & Definição do octante \\
\hline Norte & $337,5 \leq$ azimute $<22,5$ \\
\hline Nordeste & $22,5 \leq$ azimute $<67,5$ \\
\hline Leste & $67,5 \leq$ azimute $<112,5$ \\
\hline Sudeste & $112,5 \leq$ azimute $<157,5$ \\
\hline Sul & $157,5 \leq$ azimute $<202,5$ \\
\hline Sudoeste & $202,5 \leq$ azimute $<247,5$ \\
\hline Oeste & $247,5 \leq$ azimute $<292,5$ \\
\hline Noroeste & $292,5 \leq$ azimute $<337,5$ \\
\hline
\end{tabular}

Fonte: Os autores.

O arquivo climático utilizado, com 13 anos de dados horários, foi fornecido pelo Instituto Nacional de Meteorologia - INMET. Estes dados foram registrados pela estação meteorológica automática de superfície A001-Brasília-DF, no período 2001-2013 (INMET, 2013).

Para a aplicação do método da ASHRAE 160:2009 edifício analisado foi classificado na categoria de edifícios com altura de $15 \mathrm{~m}$ a $20 \mathrm{~m}$. Neste caso, 1,2 é o valor do coeficiente $\mathrm{Fe}$ - Fator de Exposição à chuva, aplicado na Equação 6. Para a definição do coeficiente Fd - Fator de Deposição da chuva, foi atribuído o valor de 1,0, considerando que o edifício possui cobertura plana, sem presença de beiral ou marquise. Quanto à topografia, o terreno foi considerado do tipo exposição média.

No caso do método da ISO 15927-3:2009, o edifício está inserido em uma área urbana correspondente à categoria 4 , isto é, pelo menos $15 \%$ da superfície tem edifícios com uma altura superior a $15 \mathrm{~m}$. Neste caso, foi atribuído o Fator de Rugosidade $\mathrm{Cr}=0,90$. Para a definição do Fator de Topografia $\mathrm{CT}=1$, foi considerada a situação topográfica da região analisada com declividade menor que 0,05. Como existe a possibilidade de ocorrência de ventos convergentes na direção das paredes da edificação, foi definido o Fator de Obstrução $\mathrm{O}=1$. Foi adotado o Fator de Parede $\mathrm{W}=0,5$, visto que a edificação em análise possui mais de 3 pisos; cobertura plana; e a parte do 
edifício em análise corresponde à região mais crítica e exposta à chuva dirigida (parte superior da edificação).

Para a aplicação da Equação 7, referente ao método WUFI Pro 5.3:2013, foi considerado R2 = 0,2, que é o coeficiente para a categoria Edifício Alto, cuja parte superior (topo da platibanda) está a $20 \mathrm{~m}$ de altura. Para a definição do coeficiente de transferência à superfície da chuva dirigida, foi considerado o fator de redução $\mathrm{Fr}=0,7$, correspondente a $70 \%$ da água incidente na fachada, adequado ao elemento construtivo cuja inclinação é igual a 90o.

Para alimentar o programa WUFI Pro 5.3 (IBP, 2013), foi utilizado o arquivo climático TMY (Typical Meteorological Year) (RORIZ, 2012) para a cidade de Brasília.

\section{Resultados e discussões}

Os resultados dos estudos descritivos e analíticos desenvolvidos, aplicados ao contexto urbano da cidade de Brasília, são aqui apresentados. As Figuras 3, 4 e 5 ilustram os resultados mensais das quantificações de água de chuva obtidas por meio da aplicação dos métodos semiempíricos. Analisando a intensidade da chuva dirigida obtida por meio dos três diferentes métodos de quantificação, observa-se diferenças bastante significativas nos resultados ilustrados nos gráficos e sintetizados na Figura 2.

Essa discrepância entre os valores ocorre porque cada método considera diferentes coeficientes de representação das características arquitetônicas e das condições de exposição do edifício em relação ao lugar (topografia, rugosidade, altura e geometria do edifício, tipo de cobertura, proteções, etc.).
Figura 2 - Comparação entre três métodos de quantificação de chuva dirigida (I/m2) para a cidade de Brasília

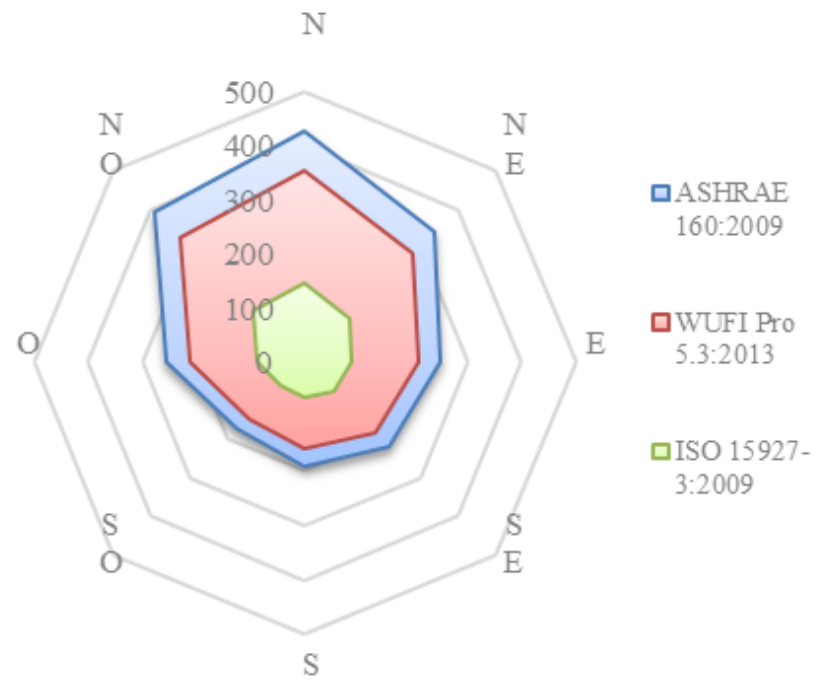

Fonte: Os autores.

O método do WUFI Pro 5.3:2013 considera uma única condição, que está relacionada ao fator de parede, isto é, a altura da região da parede onde se está estudando a incidência da chuva dirigida. Para a pior condição, ou seja, para região do edifício com $20 \mathrm{~m}$ de altura, o WUFI Pro 5.3:2013 considera um valor igual a 0,2 enquanto o método da ASHRAE 160:2009 considera o valor 1,0 e o método da ISO 15927-3:2009 considera o valor 0,5. Portanto, constata-se que o parâmetro mencionado é bastante variável entre os métodos.

Quanto às condições urbanas locais onde o edifício está inserido, o WUFI Pro 5.3:2013 não estabelece nenhum fator, enquanto a ISO 15927-3:2009 estabelece quatro fatores e a ASHRAE 160:2009 considera dois fatores.

Figura 3 - Resultado da quantificação de chuva dirigida (l/m2) em Brasília: método ASHRAE 160:2009

Intensidade de chuva dirigida (I/m2) pelo método ASHRAE 160:2009

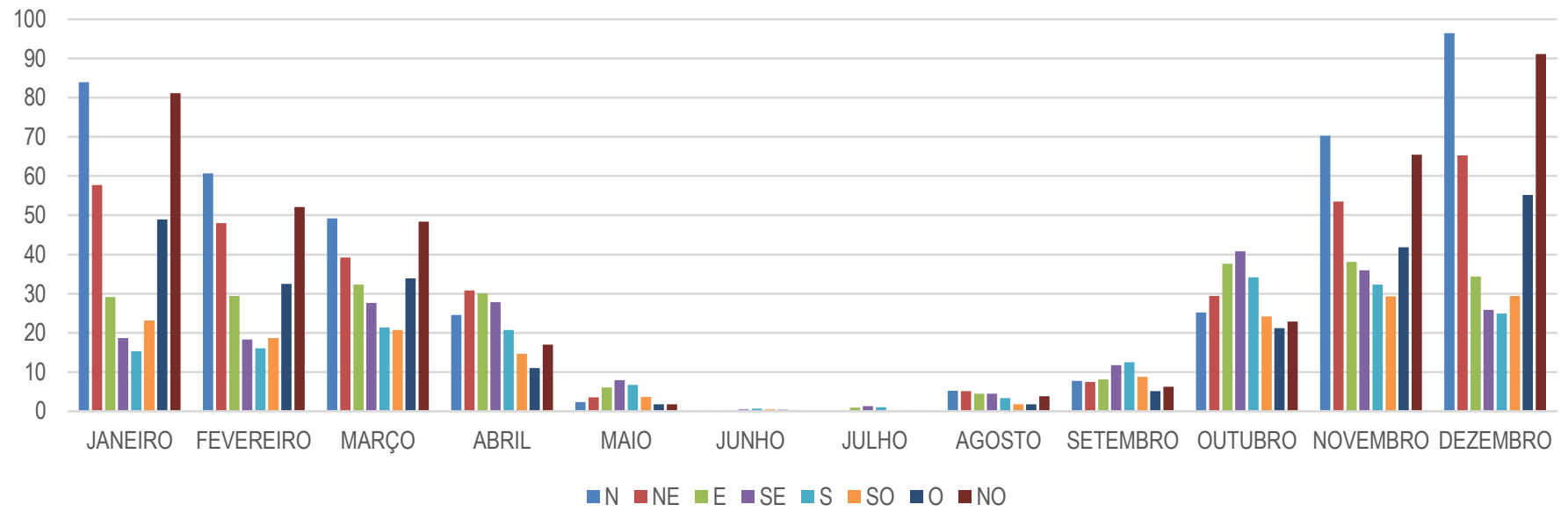

Fonte: Os autores. 
Figura 4 - Resultado da quantificação de chuva dirigida (I/m2) em Brasília: método WUFI Pro 5.3:2013 (com fator de redução de 0,7)

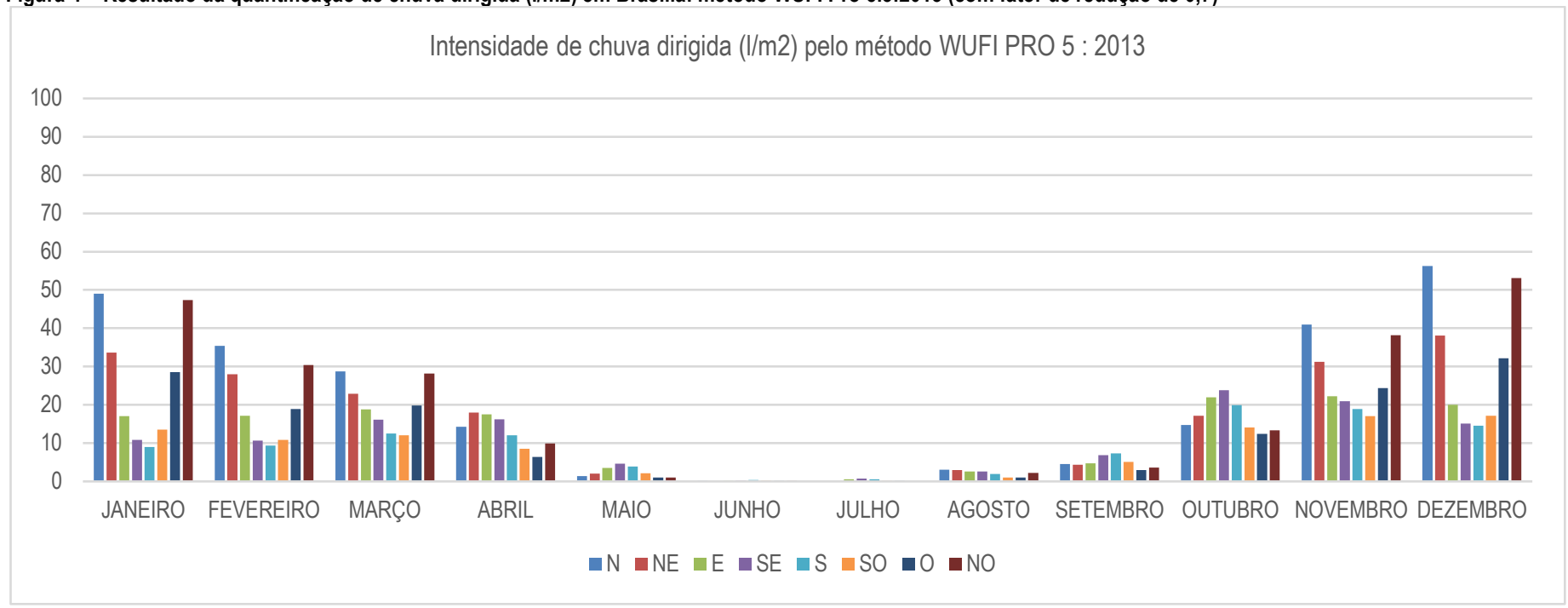

Fonte: Os autores.

Figura 5- Resultado da quantificação de chuva dirigida (I/m2) em Brasília: método ISO 15927-3:2009 Intensidade de chuva dirigida (I/m2) pelo método WUFI PRO 5 : 2013

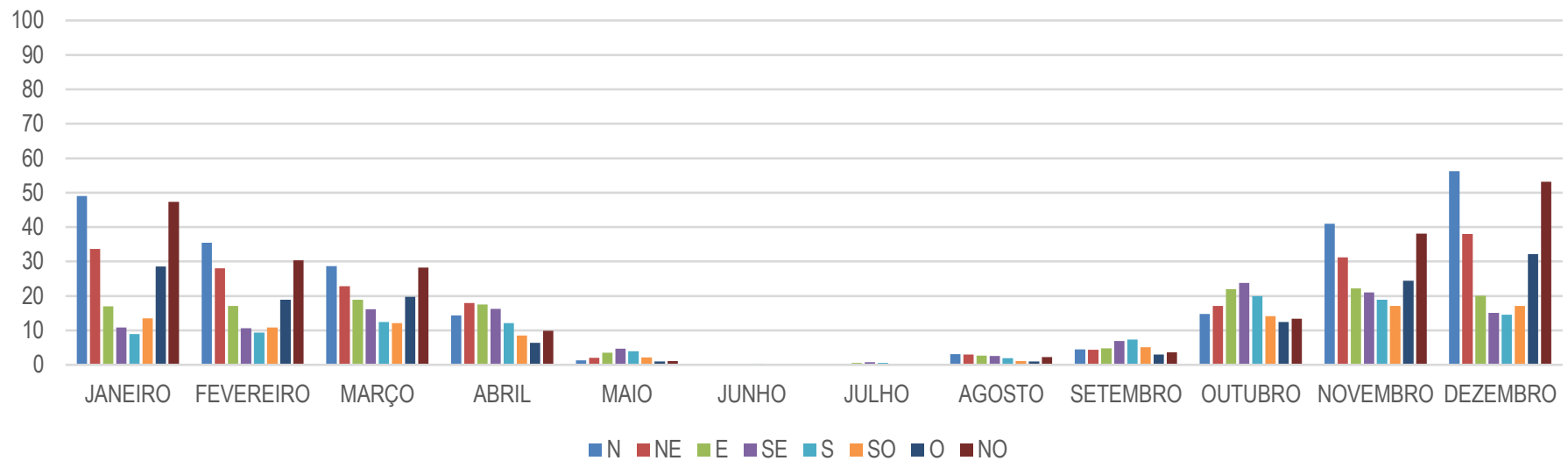

Fonte: Os autores.

Tanto o método da ISO 15927-3:2009 como o método da ASHRAE 160:2009 consideram a constante de proporcionalidade que relaciona a chuva dirigida em uma situação de campo aberto.

A ASHRAE 160:2009 considera, em um único fator, o ajuste necessário para as condições de topografia, rugosidade e exposição do edifício, relacionando os valores com a altura do edifício e com três categorias de exposição. Por outro lado, a ISO 15927-3:2009 adota quatro fatores de ajustes, pois considera separadamente os valores para cada um dos fatores mencionados, resultando em menores montantes calculados.

Importante esclarecer que, nesta análise comparativa entre os três métodos, não foi considerado o fator de redução de 0,7 utilizado nos estudos de simulação higrotérmica, para definir quanto de água líquida está disponível para umedecer a fachada, pois o objetivo foi verificar os montantes de chuva que podem atingir a superfície.
A aplicação dos três principais métodos semi-empíricos (ISO 15927-3:2009, ASHRAE 160:2009 e WUFI Pro 5.3:2013) permitiu quantificar a intensidade de água de chuva que é projetada na fachada em cada mês do ano, identificando qual orientação de fachada é mais exposta à umidificação pela ação da chuva dirigida.

Os resultados mensais representados nas Figuras 3, 4 e 5 mostram que a maior intensidade de chuva dirigida ocorre no mês de dezembro, seguido pelos meses de janeiro, novembro, fevereiro, março e outubro (meses do período chuvoso). No período seco (abril a setembro), a intensidade de chuva dirigida é bastante baixa, principalmente nos meses de maio, junho, julho e agosto.

Quanto à orientação mais exposta à chuva dirigida, a fachada Norte é aquela que recebe a maior quantidade de chuva ao longo do ano, seguida pela fachada Noroeste. Isso é explicado, considerando que as direções Noroeste e 
Norte estão entre aquelas onde ocorrem os ventos predominantes associados aos eventos de precipitação.

As orientações de fachada mais atingidas pela chuva dirigida independem do método (são as mesmas em todos os métodos), pois são resultado da ação combinada da precipitação e vento (direção e velocidade).

Para balizar a seleção de qual método é mais representativo para as condições brasileiras, em especial para o contexto estudado, é necessário a realização de um programa experimental in situ que permite averiguar qual dos métodos representa melhor correlação com os valores de medições feitas em situação real.

\section{Considerações finais}

Os estudos descritivos e analíticos aplicados para o contexto urbano da cidade de Brasília-DF, permitiram avaliar três métodos semi-empíricos para quantificação da intensidade de chuva dirigida projetada na fachada. Por meio da comparação, foi possível constatar diferenças significativas nos resultados obtidos.

Os métodos semi-empíricos quantificam a intensidade de chuva dirigida a partir de coeficientes destinados a ajustar as condições em campo aberto para as condições locais das fachadas, de acordo com o contexto urbano onde o edifício está inserido, inclusive levando em consideração as características do edifício.

Os resultados obtidos por meio desses métodos variam em decorrência do coeficiente de chuva dirigida $\alpha$ que corresponde ao conjunto de parâmetros e constantes adotados para ajustar as condições urbanas e as características da edificação.

Importante salientar que a diferença entre os métodos resulta em quantidades diferentes de chuva dirigida porque cada método adota diferentes coeficientes de chuva dirigida, cabendo ao usuário decidir qual dos métodos melhor representará a condição urbana e as variáveis relacionadas ao edifício em estudo.

Conclui-se que o método da ASHRAE 160:2009 é aquele que quantifica o maior volume de água projetada na fachada. Comparando os resultados, constata-se que os montantes calculados pelo método WUFI Pro 5.3 (IBP, 2013) correspondem a $83 \%$ daqueles obtidos pelo método recomendado pela ASHRAE 160:2009, enquanto que os valores calculados pelo método da ISO 15927-3:2009 correspondem a cerca de $35 \%$ daqueles obtidos pelo método da ASHRAE 160:2009.

\section{Referências}

ANSI/ASHRAE - AMERICAN NATIONAL STANDARDS INSTITUTE \& AMERICAN SOCIETY OF HEATING, REFRIGERATING AND AIR-CONDITIONING ENGINEERS. ANSI/ASHRAE 160 - Criteria for Moisture-Control Design Analysis in Buildings. Atlanta, 2009.

BAUER, E. Resistência a penetração da chuva em fachadas de alvenaria de materiais cerâmicos: uma análise de desempenho. 1987. Dissertação (Mestrado em Engenharia Civil) - Universidade Federal do Rio Grande do Sul. Porto Alegre.

BLOCKEN, B.; ABUKU, M.; NORE, K.; BRIGGEN, P.M.; SCHELLEN, H.L.; THUE, J.V.; ROEL, S. S.; CARMELIET, J. Intercomparison of wind-driven rain deposition models based on two case studies with full-scale measurements. Journal of Wind Engineering and Industrial Aerodynamics, v. 99, n. 4, p.448-459, abr. 2011. doi: https://doi.org/10.1016/j.jweia.2010.11.004

BLOCKEN, B.; CARMELIET, J. A review of wind-driven rain research in building science. Journal of Wind Engineering and Industrial Aerodynamics, v. 92, n. 13, p. 1079-1130, nov. 2004. doi: https://doi.org/10.1016/j.jweia.2004.06.003

BLOCKEN, B.; CARMELIET, J. Overview of three state-of-the-art wind-driven rain assessment models and comparison based on model theory. Building and Environment, v. 45, n. 3, p. 691-703, mar. 2010. doi: https://doi.org/10.1016/j.buildenv.2009.08.007

BLOCKEN, B.; DEROME, J.; CARMELIET, J. Rainwater runoff from building facades: a review. Building and Environment, v. 60, p. 339-361, Jul. 2013. doi: https://doi.org/10.1016/j.buildenv.2012.10.008

BSI - BRITISH STANDARD INSTITUTION. BS 8104: Code of practice for assessing exposure of walls to wind driven rain. London, 1992.

BSI - BRITISH STANDARD INSTITUTION. BS EN ISO 15927-3: Hygrothermal performance of buildings - Calculation and presentation of climatic data. Part 3: Calculation of a driving rain index for vertical surfaces from hourly wind and rain data. BS EN ISO, 2009. 
BSI - BRITISH STANDARD INSTITUTION. BS ISO 15686-7: Buildings and constructed assets — Service life planning — Part

7: Performance evaluation for feedback of service life data from practice. London, 2006.

CHAND, I.; BHARGAVA, P.K. Estimation of driving rain index for India. Building and Environment, v. 37, n. 5, p. 549-554, mai. 2002. doi: https://doi.org/10.1016/S0360-1323(01)00057-9

ESTRELA, P. H. T. Determinação e Análise de Índices de Exposição à Chuva Dirigida para 400 Estações Brasileiras: Dados de 1995 a 2010. 2010.Trabalho de Conclusão de Curso (Graduação em Engenharia Civil) - Instituto Tecnológico de Aeronáutica, São José dos Campos.

FREITAS, A. S. S. L. A. Avaliação do Comportamento Higrotérmico de Revestimentos Exteriores de Fachadas devido à Acção da Chuva Incidente. 2011. Dissertação (Mestre em Engenharia Civil) - Universidade do Porto, Porto.

GIONGO, M.; PADARATZ, I. J.; LAMBERTS, R. Determinação da exposição à chuva dirigida em Florianópolis, SC: índices de chuva dirigida e métodos semi-empíricos. Ambiente Construído, Porto Alegre, v. 11, n. 1, p. 7-23, jan./mar. 2011. doi:http://dx.doi.org/10.1590/S1678-86212011000100002

GIONGO, M. Análise do Nível de Exposição das Edificações à Chuva Dirigida para Florianópolis. 2007. Dissertação (Mestrado em Engenharia Civil) - Universidade Federal de Santa Catarina, Florianópolis.

HOPPESTAD, S. Slagregn i Norge (in Norwegian). Norwegian Building Research Institute, rapport 13, Oslo, 1955.

IBP - FRAUNHOFER INSTITUTE FOR BUILDING PHYSICS -. WUFI Pro 5.3. Holzkirchen, Germany, 2013.

INMET - INSTITUTO NACIONAL DE METEOROLOGIA. Arquivos de dados horários A001- Brasília-DF. Banco de Dados Meteorológicos para Ensino e Pesquisa (BDMEP), 2013.

KAZMIERCZAK, C. S. Desempenho de Alvenarias de Materiais Cerâmicos à Penetração de Água da Chuva: Uma Análise de Fatores Condicionantes. 1989. Dissertação (Mestrado em Engenharia Civil) - Universidade Federal do Rio Grande do Sul, Porto Alegre.

KRPAN, R. Wind-driven Rain on Buildings in Metro Vancouver: Parameters for Rain Penetration Testing of Window Assemblies. 2013. Thesis (PhD in Applied Science) - Building, Civil and Environmental Engineering at Concordia University, Canada.

LACY, R.E.; SHELLARD, H.C. An index of driving rain. The Meteorological Magazine, Great Britain, v. 91, n. 1080, p. 177184, jul. 1962.

LACY, R. Driving-rain maps and the onslaught of rain on buildings. Building Research Station. Garston, England: Department of Scientific and Industrial Research, 1965.

LACY, R. E. Survey of Meteorological Information for Architecture and Building. (S.l): Building Research Station, Department of the Environment, 1972.

LIMA, M. G., MORELLI, F. Mapa Brasileiro de Chuva Dirigida - Algumas Considerações. In: SIMPÓSIO BRASILEIRO TECNOLOGIA DE ARGAMASSAS, 7, 2005, Florianópolis. Anais... Florianópolis: ANTAC, 2005. p. 620-634.

LIMA, M. G.; ESTRELA, P.H.T. Determinação e análise de índices de exposição à chuva dirigida para São José dos Campos - SP com base em dados dos anos de 2008 e 2009. In: ENCONTRO NACIONAL DE TECNOLOGIA DO AMBIENTE

CONSTRUÍDO, 13, 2010. Anais ... Canela-RS: ANTAC, 2010. p. 1-9.

MARSH, P. Air \& Rain Penetration of Buildings. New York: The Construction Press, 1977.

MELO JÚNIOR, C. M.; CARASEK, H. Índices de chuva dirigida direcional e análise do nível de umedecimento em fachadas de edifício em multipavimentos em Goiânia, GO. Ambiente Construído, Porto Alegre, v. 11, n. 3, p. 23-37, jul./set. 2011.

PEREZ, A. R. Umidade nas edificações. 1986. Dissertação (Mestrado em Engenharia Civil) - Escola Politécnica da Universidade de São Paulo, São Paulo. 
RORIZ, M. Correções nas Irradiâncias e Iluminâncias dos arquivos EPW da Base ANTAC. Grupo de Trabalho sobre Conforto e Eficiência Energética de Edificações. ANTAC - Associação Nacional de Tecnologia do Ambiente Construído. São Carlos - SP, 2012. Disponível em: http://www.labeee.ufsc.br/downloads/arquivos-climaticos/formato-epw. Acesso em: setembro de 2013.

SILVA, A.C.S.B.; GIRALT, R.P. Estabelecimento de um índice de chuva dirigida para Porto Alegre. In: ENCONTRO NACIONAL DE TECNOLOGIA DO AMBIENTE CONSTRUÍDO, 6, 1995, Rio de Janeiro. Anais... Rio de Janeiro: ANTAC, 1995. p. 605-610.

STRAUBE, J. BSD-148: Simplified Prediction of Driving Rain on Buildings: ASHRAE 160P and WUFI 4.0. Building Science Corporation, Building Science Digest, 2010. Disponível em: < https://buildingscience.com/documents/digests/bsd-148-wufisimplified-driving-rain-prediction >. Acesso em: 18.06.2018.

ZANONI, V. A. G.; SÁNCHEZ, J.M. M.; BAUER, E.; AMORIM, C.N.D. Matriz de análise do índice de chuva dirigida (ICD) para a cidade de Brasília-DF. Paranoá: cadernos de arquitetura e urbanismo, v.11, p. 67-76, 2014.

doi:http://dx.doi.org/10.18830/issn.1679-0944.n11.2014.12085

ZANONI, V.A.G. Influência dos agentes climáticos de degradação no comportamento higrotérmico de fachadas em Brasília. 2015.Tese (Doutorado em Arquitetura e Urbanismo) - Faculdade de Arquitetura e Urbanismo, Universidade de Brasília, Brasília.

\section{${ }^{1}$ Vanda Alice Garcia Zanoni}

Engenheira Civil. Doutora. Endereço postal: Campus Universitário Darcy Ribeiro, Brasília, Distrito Federal, Brasil, CEP: 70910900 .

\section{José Manoel Morales Sanchez}

Engenheiro Civil. Doutor. Endereço postal: Campus Universitário Darcy Ribeiro, Brasília, Distrito Federal, Brasil, CEP: 70910-900

\section{${ }^{3}$ Elton Bauer}

Engenheiro Civil. Doutor. Endereço postal: Campus Universitário Darcy Ribeiro, Brasília, Distrito Federal, Brasil, CEP: 70910900. 